\title{
On links with cyclotomic Jones polynomials
}

\author{
ABHIJIT CHAMPANERKAR \\ ILYA KOFMAN
}

\begin{abstract}
We show that if $\left\{L_{n}\right\}$ is any infinite sequence of links with twist number $\tau\left(L_{n}\right)$ and with cyclotomic Jones polynomials of increasing span, then $\lim \sup \tau\left(L_{n}\right)=\infty$. This implies that any infinite sequence of prime alternating links with cyclotomic Jones polynomials must have unbounded hyperbolic volume. The main tool is the multivariable twist-bracket polynomial, which generalizes the Kauffman bracket to link diagrams with open twist sites.
\end{abstract}

$57 \mathrm{M} 25 ; 26 \mathrm{C} 10$

\section{Introduction}

In [2], we showed that the Mahler measure of the Jones polynomial converges under twisting for any link. This is consistent with the convergence of hyperbolic volume under the corresponding Dehn surgery. In this note, we consider knots or links whose Jones polynomials are distinct but with constant Mahler measure equal to one, which we call cyclotomic. All known constructions of infinite sequences of hyperbolic knots or links with the same Jones polynomial (eg, Kanenobu [5; 4] and Watson [11]) require twisting at finitely many sites, and hence all such families have bounded volume. In contrast, any infinite sequence of hyperbolic alternating links with cyclotomic Jones polynomials must have unbounded volume by Corollary 1.2 below.

For any link diagram $L$, two crossings are in the same twist class if there is a simple closed curve that transversally intersects the projection of $L$ only at the two crossing points and encloses only adjacent bigons of the diagram. The twist number $\tau(L)$, originally defined by Lackenby in [6], is the number of twist classes of crossings of $L$. In general, for any collection of links with bounded twist number, their Jones polynomials have bounded Mahler measure (Silver, Stoimenow and Williams [10]); see Proposition 3.5 below.

Theorem 1.1 If $\{L(m)\}$ is any sequence of links with cyclotomic Jones polynomials $V_{L(m)}(t)$, and $\lim _{m \rightarrow \infty} \operatorname{span}\left(V_{L(m)}(t)\right)=\infty$, then $\lim \sup \tau(L(m))=\infty$. 
Our theorem, together with Theorem 1 of [6], implies the following corollary, where $\operatorname{Vol}\left(S^{3} \backslash L\right)$ denotes hyperbolic volume:

Corollary 1.2 If $\{L(m)\}$ is any infinite sequence of distinct prime alternating links with cyclotomic Jones polynomials, then $\lim \sup \operatorname{Vol}\left(S^{3} \backslash L(m)\right)=\infty$.

A simple example that illustrates Theorem 1.1 is the sequence of connect sums of the figure-eight knot. Prime knots with cyclotomic Jones polynomials are relatively rare: there are 17 such knots in 1.7 million knots with up to 16 crossings (see Remark 1 of [2]). We do not know such an infinite sequence of hyperbolic knots, although the simplest hyperbolic knots have Jones polynomials with unusually small Mahler measure [3].

The main tool in the proof is our generalization of the Kauffman bracket polynomial for links to the twist-bracket polynomial $P\left(A, x_{1}, \ldots, x_{k}\right)$ for link diagrams with $k$ open twist sites. After normalizing, we obtain a regular isotopy invariant for these diagrams, which are sometimes called 2-strand block diagrams. Details and examples are given in the next section.

The twist-bracket polynomial is a special case of the multivariable polynomial introduced in [2], which was defined using minimal central idempotents in the TemperleyLieb algebra. By twisting on only two strands at every twist site, we obtain an explicit formula in terms of the Kauffman bracket. In general, a link diagram with $k$ open twist sites gives rise to a $k$-linear form on the Temperley-Lieb algebra, and the twist-bracket polynomial is naturally associated to this form. For details, see $[2 ; 11]$.

Acknowledgments The authors acknowledge support from the National Science Foundation under an FRG grant (DMS-0455978 and DMS-0456227, respectively).

\section{The twist-bracket polynomial}

For any link diagram $L$, its Kauffman bracket $\langle L\rangle \in \mathbb{Z}\left[A^{ \pm 1}\right]$ equals the Jones polynomial $V_{L}(t)$, up to a monomial that depends on the writhe $w(L)$ of the diagram: If $t=A^{-4}, V_{L}(t)=(-A)^{-3 w(L)}\langle L\rangle$.

A wiring diagram is a regular 4-valent planar graph with two kinds of vertices, called twist sites, which are oriented either horizontally or vertically. We say that a link diagram $L$ can be obtained from a wiring diagram $\tilde{L}$ if $L$ is realized by inserting a twist class of crossings at every twist site of $\widetilde{L}$, according to its horizontal or vertical orientation. For any $L$, there exists $\widetilde{L}$ with $v(\widetilde{L})=\tau(L)$ such that $L$ can be obtained 
from $\widetilde{L}$. If all but $k$ twist sites have crossings inserted, we will say that the diagram $\tilde{L}$ has $k$ open twist sites. A twist site will be called nugatory if the isotopy class of the link does not depend on how many crossings are inserted there.

Let $\widetilde{L}$ be a diagram with $k$ open twist sites. For $\mathbf{s} \in\{0,1\}^{k}$, let $\widetilde{L}_{\mathbf{s}}$ be obtained as follows: If the $i$-th twist site is oriented vertically, insert )( when $s_{i}=0$, and $\asymp$ when $s_{i}=1$. Otherwise, insert $\asymp$ when $s_{i}=0$, and $)\left(\right.$ when $s_{i}=1$. For $\mathbf{n} \in \mathbb{Z}^{k}$, let $L_{\mathbf{n}}$ be the diagram obtained from $\widetilde{L}$ by inserting at the $i$-th twist site: $n_{i}$ half-twists on 2 strands, which is $\left|n_{i}\right|$ crossings, with sign according to right- or left-handed twisting. To be precise, if $n_{i}=1$, we insert the crossing whose $A$-smoothing corresponds to $s_{i}=0$. If $n_{i}=0$, we insert $)\left(\right.$ or $\asymp$ as in $s_{i}=0$.
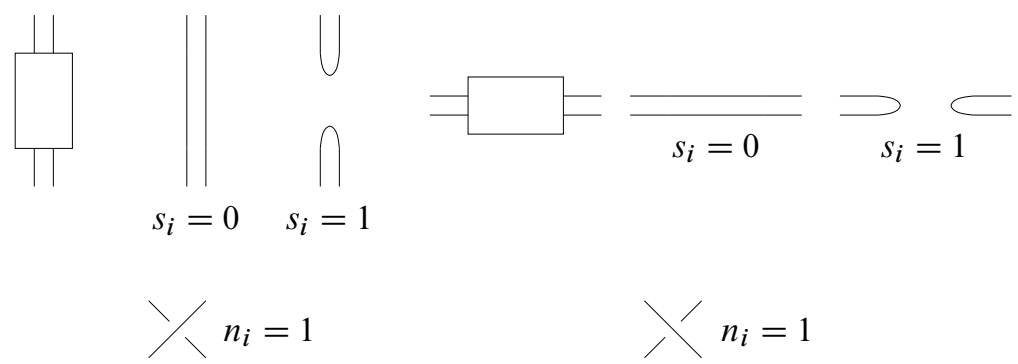

Let $|\mathbf{s}|=\sum_{i=1}^{k} s_{i}$ and $\sigma(\mathbf{n})=\sum_{i=1}^{k} n_{i}$. Let $\delta=-A^{2}-A^{-2}$.

Definition 1 For any $\widetilde{L}$ with $k$ open twist sites, we define its twist-bracket by:

$$
P\left(A, x_{1}, \ldots, x_{k}\right)=\sum_{\mathbf{s} \in\{0,1\}^{k}}\left(\prod_{i=1}^{k}\left(x_{i}-1\right)^{s_{i}} \delta^{1-s_{i}}\right)\left\langle\widetilde{L}_{\mathbf{s}}\right\rangle
$$

From the definition, $P\left(A, x_{1}, \ldots, x_{k}\right) \in \mathbb{Z}\left[A^{ \pm 1}, x_{1}, \ldots, x_{k}\right]$.

Proposition 2.1 If $\tilde{L}$ is any diagram with $k$ open twist sites, then

$$
\delta^{k}\left\langle L_{\mathbf{n}}\right\rangle=A^{\sigma(\mathbf{n})} P\left(A,\left(-A^{-4}\right)^{n_{1}}, \ldots,\left(-A^{-4}\right)^{n_{k}}\right) .
$$

Proof First, suppose $\tilde{L}$ has only one open twist site, which we orient vertically. We claim that, similar to Proposition 3.3 [2],

$$
\delta\left\langle L_{n}\right\rangle=A^{n}\left(\delta\left\langle L_{)}\right\rangle+\left(\left(-A^{-4}\right)^{n}-1\right)\left\langle L_{\asymp}\right\rangle\right) .
$$

If $\left\{\mathbf{1}, e_{1}\right\}$ is the $T L_{2}$ basis, $p_{0}=\mathbf{1}-e_{1} / \delta$ is the Jones-Wenzl idempotent, and $p_{1}=\mathbf{1}-$ $p_{0}$ is the orthogonal idempotent. Let $\rho: B_{2} \rightarrow T L_{2}$ be given by $\rho\left(\sigma_{1}\right)=A \mathbf{1}+A^{-1} e_{1}$. 
Following the proof of Proposition 3.3 [2], with $\Delta=\sigma_{1}, \rho(\Delta)=A p_{0}-A^{-3} p_{1}$, hence $\rho\left(\Delta^{n}\right)=A^{n} p_{0}+(-1)^{n} A^{-3 n} p_{1}$.

$$
\begin{aligned}
\rho\left(\Delta^{n}\right) & =A^{n}\left(p_{0}+(-1)^{n} A^{-4 n} p_{1}\right) \\
& =A^{n}\left(\mathbf{1}-\frac{e_{1}}{\delta}+(-1)^{n} A^{-4 n} \frac{e_{1}}{\delta}\right) \\
& \left.=A^{n}\left(\mathbf{1}+\frac{\left((-1)^{n} A^{-4 n}-1\right)}{\delta} e_{1}\right)\right)
\end{aligned}
$$

Using the bilinear form on $T L_{2},\left\langle L_{n}\right\rangle=\left\langle\widetilde{L}, \Delta^{n}\right\rangle$, so (1) follows.

If we repeatedly apply (1) at each twist site, we obtain:

$$
\delta^{k}\left\langle L_{\mathbf{n}}\right\rangle=A^{\sigma(\mathbf{n})} \sum_{\mathbf{s} \in\{0,1\}^{k}}\left(\prod_{i=1}^{k}\left(\left(-A^{-4}\right)^{n_{i}}-1\right)^{s_{i}} \delta^{1-s_{i}}\right)\left\langle\tilde{L}_{\mathbf{s}}\right\rangle
$$

Example 1 Double twist links $L(m, n)$ are obtained from a wiring diagram $\widetilde{L}$ with two twist sites such that $\left\langle\widetilde{L}_{(0,0)}\right\rangle=\left\langle\widetilde{L}_{(1,1)}\right\rangle=1$ and $\left\langle\widetilde{L}_{(0,1)}\right\rangle=\left\langle\widetilde{L}_{(1,0)}\right\rangle=\delta$. We get $P(A, x, y)=\delta^{2}(x+y-1)+(x-1)(y-1)$.

Example 2 2-bridge links $L\left(n_{1}, \ldots, n_{k}\right)$ are obtained from a wiring diagram $\widetilde{L}^{k}$ that depends on the parity of $k$ (see eg, Lickorish [8, Figure 1.8]). $\tilde{L}^{k}$ satisfies the following recurrence: $\widetilde{L}_{(\mathrm{s}, 1)}^{k}=\widetilde{L}_{\mathbf{s}}^{k-1}, \widetilde{L}_{(\mathrm{s}, 0,0)}^{k}=\widetilde{L}_{\mathbf{s}}^{k-2}, \widetilde{L}_{(\mathbf{s}, 1,0)}^{k}=\bigcirc \cup \widetilde{L}_{\mathbf{s}}^{k-2}$. If $P_{k}\left(A, x_{1}, \ldots, x_{k}\right)$ is the twist-bracket for $\widetilde{L}^{k}$, then $P_{k}$ satisfies the following recurrence:

$$
P_{k}=\left(x_{k}-1\right) P_{k-1}+\delta^{2} x_{k-1} P_{k-2}
$$

with $P_{1}=\delta^{2}+\left(x_{1}-1\right)$, which is the twist-bracket for $(2, \mathrm{n})$ torus links, and $P_{2}=$ $\delta^{2}\left(x_{1}+x_{2}-1\right)+\left(x_{1}-1\right)\left(x_{2}-1\right)$, which is the same twist-bracket as in Example 1.

Example 3 Pretzel links $\mathcal{P}\left(n_{1}, \ldots, n_{k}\right)$ are obtained from a wiring diagram $\tilde{L}$ with $k$ twist sites such that $\left\langle\tilde{L}_{\mathbf{s}}\right\rangle=\delta^{k-|\mathbf{s}|-1}$ for $\mathbf{s} \neq(1, \ldots, 1)$ and $\left\langle\widetilde{L}_{(1, \ldots, 1)}\right\rangle=\delta$. The following gives a closed formula for the bracket of any pretzel link:

$$
P\left(A, x_{1}, \ldots, x_{k}\right)=\frac{1}{\delta}\left(\prod_{i=1}^{k}\left(x_{i}-1+\delta^{2}\right)+\left(\delta^{2}-1\right) \prod_{i=1}^{k}\left(x_{i}-1\right)\right)
$$

Example 4 Kanenobu links $K(p, q)$ given in [5] are obtained from a diagram $\widetilde{L}$ with two open twist sites such that $\left\langle\widetilde{L}_{(1,0)}\right\rangle=\left\langle\widetilde{L}_{(0,1)}\right\rangle=\delta,\left\langle\widetilde{L}_{(1,1)}\right\rangle=\delta^{2}$ and $\left\langle\widetilde{L}_{(0,0)}\right\rangle=$ $\left\langle 8_{9}\right\rangle=\left\langle 4_{1}\right\rangle^{2}$, where both knot diagrams have writhe $=0$. After simplifying, we get $P(A, x, y)=\delta^{2}\left(\left\langle 8_{9}\right\rangle-1+x y\right)$. For $p+q=$ constant, the writhe is constant, so these links have the same Jones polynomial. 
Example 5 Kanenobu links $K\left(n_{1}, \ldots, n_{k}\right)$ given in Figure 9 of [4] are obtained from a diagram $\widetilde{L}$ with $k$ open twist sites such that $\left\langle\widetilde{L}_{\mathbf{s}}\right\rangle=\delta^{|\mathbf{s}|}$ for $\mathbf{s} \neq(0, \ldots, 0)$. After simplifying, we get:

$$
P\left(A, x_{1}, \ldots, x_{k}\right)=\delta^{k}\left(\left\langle\tilde{L}_{(0, \ldots, 0)}\right\rangle-1+\prod_{i=1}^{k} x_{i}\right)
$$

For $\sum n_{i}=$ constant, the writhe is constant, so these links have the same Jones polynomial.

If $\widetilde{L}$ has only one open twist site (oriented vertically), Proposition 2.1 implies

$$
\delta\left\langle L_{n}\right\rangle=A^{n} P\left(A,\left(-A^{-4}\right)^{n}\right) \quad \text { with } \quad P(A, x)=\delta\left\langle L_{)}\right\rangle+(x-1)\left\langle L_{\asymp}\right\rangle .
$$

Proposition 2.2 If $P(A, x)=x \cdot f(A)$ for some $f$, then $V_{L_{n}}(t)=V_{L)(}(t)$ for all $n$.

Proof By (2), $P(A, x)=x \cdot f(A)$ occurs only if $\delta\left\langle L_{)}\right\rangle=\left\langle L_{\asymp}\right\rangle$. This has several implications: First, by (1),

$$
\delta\left\langle L_{n}\right\rangle=A^{n}\left(-A^{-4}\right)^{n}\left\langle L_{\asymp}\right\rangle=\left(-A^{-3}\right)^{n}\left\langle L_{\asymp}\right\rangle .
$$

Second, at $A=1,\left\langle L_{\asymp}\right\rangle=(-2)\left\langle L_{)}\right\rangle$. Generally, if $\mu(L)$ is the number of components of $L$, and $c(L)$ is the number of crossings of $L$, then at $A=1$,

$$
\langle L\rangle=(-1)^{w(L)}(-2)^{\mu(L)-1}=(-1)^{c(L)}(-2)^{\mu(L)-1}
$$

Therefore, the number of components changes: $\mu\left(L_{\asymp}\right)=\mu\left(L_{)}\right)+1$. The following are possibilities for $L_{)(}$at the twist site, up to crossing changes in the rest of the link diagram:

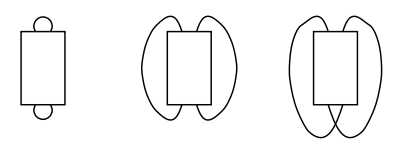

The condition $\mu\left(L_{\asymp}\right)=\mu\left(L_{)(}\right)+1$ excludes all except the first type. At such a twist site, the strands of $L_{)}$must be oriented in opposite directions:

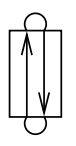

Algebraic $8 \mathcal{G}$ Geometric Topology, Volume 6 (2006) 
Therefore, $w\left(L_{n}\right)=w\left(L_{)(}\right)-n$.

$$
\begin{aligned}
V_{L_{n}}(t) & =\left(-A^{-3}\right)^{w\left(L_{n}\right)}\left\langle L_{n}\right\rangle=\left(-A^{-3}\right)^{w(L)()^{-n}}\left(-A^{-3}\right)^{n}\left\langle L_{\asymp}\right\rangle / \delta \\
& =\left(-A^{-3}\right)^{w(L)\left({ }^{)}\right.}\left\langle L_{)(}\right\rangle=V_{L_{)(}}(t)
\end{aligned}
$$

For any $\widetilde{L}$ with $k$ open twist sites, we define its normalized twist-bracket by

$$
\bar{P}_{\widetilde{L}}\left(A, x_{1}, \ldots, x_{k}\right)=\frac{1}{\delta^{k}} P_{\widetilde{L}}\left(A, x_{1}, \ldots, x_{k}\right) .
$$

The Kauffman bracket is an invariant of regular isotopy for link diagrams. We now show that the normalized twist-bracket is an invariant of regular isotopy for link diagrams with open twist sites. Two link diagrams with open twist sites will be called regularly isotopic if they are related by the following moves:

(i) Planar isotopy.

(ii) Reidemeister moves $I I$ and $I I I$, naturally augmented so that strands of the link can pass across open twist sites.

(iii) Combining and separating adjacent open twist sites:

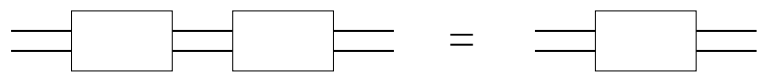

Proposition 2.3 If two link diagrams with open twist sites are regularly isotopic, then their normalized twist-brackets are equal, after possibly changing variables.

Proof The invariance of the Kauffman bracket under moves (i) and (ii) implies invariance of the twist-bracket under these moves. We now consider move (iii) for diagrams $\widetilde{L}^{1}$ and $\widetilde{L}^{2}$, which are otherwise the same. The terms of $\bar{P}_{\widetilde{L}^{1}}$ and $\bar{P}_{\widetilde{L}^{2}}$ for all $\mathbf{s}$ in the twist sites shown are given in the following table:

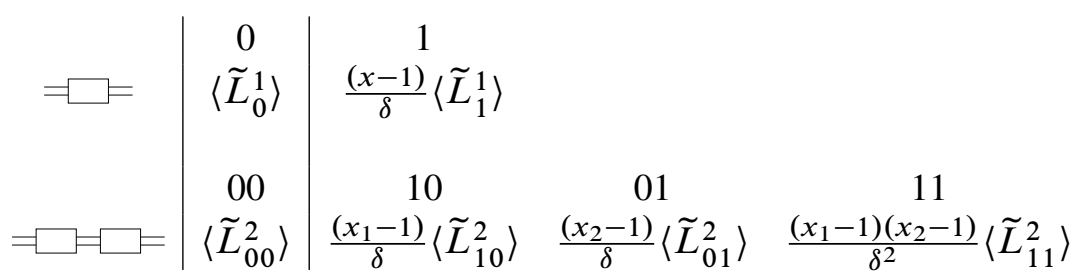

We now observe that $\left\langle\widetilde{L}_{0}^{1}\right\rangle=\left\langle\widetilde{L}_{00}^{2}\right\rangle \quad$ and $\left\langle\widetilde{L}_{1}^{1}\right\rangle=\left\langle\widetilde{L}_{10}^{2}\right\rangle=\left\langle\widetilde{L}_{01}^{2}\right\rangle=\frac{\left\langle\widetilde{L}_{11}^{2}\right\rangle}{\delta}$.

Since $\left(x_{1}-1\right)+\left(x_{2}-1\right)+\left(x_{1}-1\right)\left(x_{2}-1\right)=\left(x_{1} x_{2}-1\right)$, we have that

$$
\bar{P}_{\widetilde{L}^{1}}\left(A,\left(x_{1} x_{2}\right)\right)=\bar{P}_{\widetilde{L}^{2}}\left(A, x_{1}, x_{2}\right)=\bar{P}_{\widetilde{L}^{2}}\left(A,\left(x_{1} x_{2}\right), 1\right)=\bar{P}_{\widetilde{L}^{2}}\left(A, 1,\left(x_{1} x_{2}\right)\right) .
$$


Hence, after possible variable changes, the normalized twist-bracket $\bar{P}_{\widetilde{L}}$ is an invariant of $\tilde{L}$ up to regular isotopy.

Lemma 2.4 Let $\widetilde{L}$ be any diagram with $k$ open twist sites, obtained as above from a wiring diagram with $N$ twist sites by inserting $n_{j}$ crossings at the $j$-th twist site for each $k<j \leq N$. If $\xi=e^{2 \pi i / 6}$ then:

$$
P\left(\xi, x_{1}, \ldots, x_{k}\right)=\prod_{i=1}^{k} x_{i} \prod_{j=k+1}^{N}\left(-\xi^{-3}\right)^{n_{j}}
$$

Proof Since $\xi^{4}+\xi^{2}+1=0$, if $A=\xi$ then $\delta=-A^{2}-A^{-2}=1$. If $\widetilde{L}^{\prime}$ is a wiring diagram, then $\widetilde{L}_{\mathbf{s}}^{\prime}$ is a collection of loops, so $\left\langle\tilde{L}_{\mathbf{s}}^{\prime}\right\rangle=\delta^{\mu-1}=1$. Therefore:

$$
P\left(\xi, x_{1}, \ldots, x_{N}\right)=\sum_{\mathbf{s} \in\{0,1\}^{N}}\left(\prod_{i=1}^{N}\left(x_{i}-1\right)^{s_{i}}\right)=\prod_{i=1}^{N} x_{i}
$$

The last equality follows from the identity,

$$
\prod_{i=1}^{N}\left(X_{i}+1\right)=\sum_{\mathbf{s} \in\{0,1\}^{N}} \prod_{i=1}^{N} X_{i}^{s_{i}}
$$

with $X_{i}=x_{i}-1$. As $\tilde{L}$ is obtained from $\tilde{L}^{\prime}$ by inserting $n_{j}$ crossings at the $j-$ th twist site for each $k<j \leq N$, the result follows by Proposition 2.1.

\section{Mahler measure preliminaries}

Let $f \in \mathbb{C}\left[z_{1}^{ \pm 1}, \ldots, z_{s}^{ \pm 1}\right]$. The Mahler measure of $f$ is defined by

$$
M(f)=\exp \int_{0}^{1} \cdots \int_{0}^{1} \log \left|f\left(e^{2 \pi i \theta_{1}}, \ldots, e^{2 \pi i \theta_{s}}\right)\right| \mathrm{d} \theta_{1} \cdots \mathrm{d} \theta_{s} .
$$

If $s=1, f(z)=a_{0} z^{k} \prod_{i=1}^{n}\left(z-\alpha_{i}\right)$, then by Jensen's formula,

$$
M(f)=\left|a_{0}\right| \prod_{i=1}^{n} \max \left(1,\left|\alpha_{i}\right|\right) .
$$

Thus, for any $k \in \mathbb{Z} \backslash 0, M(f(z))=M\left(f\left(z^{k}\right)\right)$. Also, from the definition we get

$$
M\left(f\left(z_{1}, \ldots, z_{i}, \ldots, z_{s}\right)\right)=M\left(f\left(z_{1}, \ldots, \pm z_{i}, \ldots, z_{s}\right)\right) .
$$


The Mahler measure is multiplicative, $M\left(f_{1} f_{2}\right)=M\left(f_{1}\right) M\left(f_{2}\right)$, so it can be naturally extended to rational functions of Laurent polynomials.

A monic irreducible polynomial with coefficients in $\mathbb{Z}$ is called cyclotomic if all of its zeros are primitive roots of unity. Hence, the Mahler measure of a cyclotomic polynomial equals one. Multivariable polynomials whose Mahler measure equals one can be expressed in terms of cyclotomic polynomials as follows:

Lemma 3.1 (Boyd [1]) If $F \in \mathbb{Z}\left[x_{1}^{ \pm 1}, \ldots, x_{s}^{ \pm 1}\right]$, then $M(F)=1$ if and only if there are cyclotomic polynomials $\phi_{j}$ such that:

$$
F=\prod_{i=1}^{s} x_{i}^{a_{i}} \prod_{j=1}^{m} \phi_{j}\left(\prod_{i=1}^{s} x_{i}^{b_{i, j}}\right)
$$

Henceforth, we will use cyclotomic more loosely to mean constant Mahler measure equal to one.

For a vector $\mathbf{x} \in \mathbb{Z}^{s}$, let $h(\mathbf{x})=\max \left|x_{i}\right|$ and

$$
v(\mathbf{x})=\min \left\{h(\mathbf{a}) \mid \mathbf{a} \in \mathbb{Z}^{s} \backslash\{\mathbf{0}\}, \mathbf{a} \cdot \mathbf{x}=0\right\} .
$$

For example, $v\left(1, d, \ldots, d^{s-1}\right)=d$.

Lemma 3.2 (Boyd, Lawton [7]) For every $f \in \mathbb{C}\left[z_{1}^{ \pm 1}, \ldots, z_{s}^{ \pm 1}\right]$,

$$
M(f)=\lim _{\nu(\mathbf{x}) \rightarrow \infty} M\left(f\left(z^{x_{1}}, \ldots, z^{x_{s}}\right)\right)
$$

with the following useful special case: $M(f)=\lim _{d \rightarrow \infty} M\left(f\left(z, z^{d}, \ldots, z^{d^{s-1}}\right)\right)$.

We will use the following slight extension of Lemma 3.2:

Lemma 3.3 For every $f \in \mathbb{C}\left[z_{1}^{ \pm 1}, \ldots, z_{s}^{ \pm 1}\right]$ and every $\varepsilon \in\{ \pm 1\}^{s}$,

$$
M(f)=\lim _{v(\mathbf{x}) \rightarrow \infty} M\left(f\left(\left(\varepsilon_{1} z\right)^{x_{1}}, \ldots,\left(\varepsilon_{s} z\right)^{x_{s}}\right)\right) .
$$

Proof Fix $f$. Let $\psi(\mathbf{x}, \varepsilon)=M\left(f\left(\varepsilon_{1} z^{x_{1}}, \ldots, \varepsilon_{s} z^{x_{s}}\right)\right)$. By (4) and Lemma 3.2, for any $\varepsilon \in\{ \pm 1\}^{s}$,

$$
M(f)=M\left(f\left(\varepsilon_{1} z_{1}, \ldots, \varepsilon_{s} z_{s}\right)\right)=\lim _{\nu(\mathbf{x}) \rightarrow \infty} \psi(\mathbf{x}, \varepsilon) .
$$

Since there are finitely many $\varepsilon$, this implies

$$
\lim _{\nu(\mathbf{x}) \rightarrow \infty} \min _{\varepsilon} \psi(\mathbf{x}, \varepsilon)=M(f)=\lim _{\nu(\mathbf{x}) \rightarrow \infty} \max _{\varepsilon} \psi(\mathbf{x}, \varepsilon) .
$$

The result now follows by the Squeeze Theorem. 
Lemma 3.4 Let $\left\{\mathbf{x}_{m}\right\}$ be an infinite sequence of vectors in $\mathbb{Z}^{k}$. If $v\left(\mathbf{x}_{m}\right) \leq R$ for all $m$, then there exists an infinite subsequence $\left\{m_{j}\right\}$ and $\mathbf{a} \in \mathbb{Z}^{k} \backslash\{\mathbf{0}\}$ such that $\mathbf{x}_{m_{j}} \cdot \mathbf{a}=0$ for all $j$.

Proof Since $v\left(\mathbf{x}_{m}\right)$ is a bounded nonnegative integer for all $m$, we can pass to a subsequence $\left\{m_{i}\right\}$ such that $v\left(\mathbf{x}_{m_{i}}\right)=C$ for all $i$. Hence for each $i$, there exists $\mathbf{a}_{m_{i}} \in \mathbb{Z}^{k} \backslash\{\boldsymbol{0}\}$ such that $\mathbf{a}_{m_{i}} \cdot \mathbf{x}_{m_{i}}=0$ and $h\left(\mathbf{a}_{m_{i}}\right)=C$. Thus for all $i, \mathbf{a}_{m_{i}} \in$ $[-C, C]^{k} \cap \mathbb{Z}^{k} \backslash\{\boldsymbol{0}\}$. Since there are only finitely many integer lattice points in $[-C, C]^{k}$, there exists a subsequence $\left\{\mathbf{a}_{m_{j}}\right\}$ that is constant.

If $\left\{\mathbf{x}_{m}\right\}$ is a sequence of vectors with bounded $v\left(\mathbf{x}_{m}\right)$, Lemma 3.4 allows us to pass to a subsequence and apply Lemma 3.2, though the limiting polynomial will have fewer variables.

Example 6 Let $\mathbf{x}_{m}=(1, m, 2 m+1)$. Since $(1,2,-1) \cdot(1, m, 2 m+1)=0, v\left(\mathbf{x}_{m}\right) \leq 2$. Let $P(t, x, y)$ be a 3-variable polynomial. As $v\left(\mathbf{x}_{m}\right) \leq 2$, it may not be true that $M\left(P\left(t, t^{m}, t^{2 m+1}\right)\right) \rightarrow M(P(t, x, y))$ as $m \rightarrow \infty$. Let $Q(t, x)=P\left(t, x, t x^{2}\right)$, then $Q\left(t, t^{m}\right)=P\left(t, t^{m}, t^{2 m+1}\right)$ and $v(1, m) \rightarrow \infty$ as $m \rightarrow \infty$. Now by Lemma 3.2, we can conclude that $M\left(P\left(t, t^{m}, t^{2 m+1}\right)\right) \rightarrow M(Q(t, x))$ as $m \rightarrow \infty$. The linear dependence of the components $(1, m, 2 m+1)$ gives us a limit in one fewer variable.

For $F \in \mathbb{Z}\left[x_{1}^{ \pm 1}, \ldots, x_{s}^{ \pm 1}\right], M(F) \leq\|F\|$ where $\|F\|$ denotes the $L^{2}$ norm of coefficients of $F$ (see Schinzel [9] for more details).

Proposition 3.5 Let $L$ be a link diagram with $\tau(L)=k$ then $M\left(V_{L}(t)\right) \leq 8^{k}$.

Proof As $V_{L}\left(A^{-4}\right)=(-A)^{-3 w(L)}\langle L\rangle, M\left(V_{L}(t)\right)=M(\langle L\rangle)$. Since $M(\delta)=1$, by Proposition 2.1, $M\left(V_{L}(t)\right)=M(\langle L\rangle)=M\left(P\left(A,\left(-A^{-4}\right)^{n_{1}}, \ldots,\left(-A^{-4}\right)^{n_{k}}\right)\right)$.

$$
\begin{aligned}
M\left(V_{L}(t)\right) & \leq\left\|P\left(A,\left(-A^{-4}\right)^{n_{1}}, \ldots,\left(-A^{-4}\right)^{n_{k}}\right)\right\| \leq\left\|P\left(A, x_{1}, \ldots, x_{k}\right)\right\| \\
& \leq \sum_{\mathbf{s} \in\{0,1\}^{k}}\left(\prod_{i=1}^{k}\left\|\left(x_{i}-1\right)\right\|^{s_{i}}\|\delta\|^{1-s_{i}}\right)\left\|\left\langle\tilde{L}_{\mathbf{s}}\right\rangle\right\| \\
& \leq \sum_{\mathbf{s} \in\{0,1\}^{k}}\left(\prod_{i=1}^{k} 2^{s_{i}} 2^{1-s_{i}}\right) 2^{k}=8^{k}
\end{aligned}
$$

The last inequality follows from the fact that $\left\langle\tilde{L}_{\mathbf{s}}\right\rangle=\delta^{r}$ where $0 \leq r \leq k$. 


\section{Cyclotomic Jones polynomials}

Let $L$ be any link diagram. Since $V_{L}(t)=(-A)^{-3 w(L)}\langle L\rangle$ with $t=A^{-4}$, the Mahler measure is unchanged: $M\left(V_{L}(t)\right)=M(\langle L\rangle)$. The span of a Laurent polynomial is the difference of its highest and lowest degrees, so $4 \operatorname{span}\left(V_{L}(t)\right)=\operatorname{span}(\langle L\rangle)$. Let $L_{m}$ be the diagram obtained from $L$ by inserting $m$ half-twists on 2 strands of $L$.

Proposition 4.1 Distinct $V_{L_{m}}(t)$ are cyclotomic for only finitely many $m$.

Proof We consider $L$ as having one open twist site, with the original link given by $L_{)(}$. By $(2), \delta\left\langle L_{m}\right\rangle=A^{m} P\left(A,\left(-A^{-4}\right)^{m}\right)$, so then

$$
M\left(V_{L_{m}}(t)\right)=M\left(\left\langle L_{m}\right\rangle\right)=M\left(P\left(A,\left(-A^{-4}\right)^{m}\right)\right) .
$$

If $M\left(V_{L_{m}}(t)\right)=1$ for infinitely many $m$ then, after passing to a subsequence, $M(P(A, x))=\lim _{m \rightarrow \infty} M\left(P\left(A, A^{-4 m}\right)\right)=1$ by Lemma 3.3. Hence by Lemma 3.1, $P(A, x)$ equals up to monomials the product of cyclotomic polynomials evaluated at monomials in $A$ and $x$. Since the Jones polynomials vary with $m$, by Proposition 2.2, $P(A, x) \neq x \cdot f(A)$ for any $f$. By (2), $P(A, x)$ is linear in $x$, so there is exactly one cyclotomic linear factor evaluated at $A^{r} x$. Hence,

$$
P(A, x)=\left(1 \pm A^{r} x\right) A^{\alpha} \prod_{j=1}^{\ell} \phi_{j}\left(A^{m_{j}}\right) \quad \Rightarrow \quad \frac{\partial P}{\partial x}(A, x)= \pm A^{r} P(A, 0) .
$$

Therefore by (2), $\left\langle L_{\asymp}\right\rangle= \pm A^{r}\left(\delta\left\langle L_{)(}\right\rangle-\left\langle L_{\asymp}\right\rangle\right)$.

At $A=1$, the equation above reduces to

$$
\left\langle L_{\asymp}\right\rangle= \pm\left(-2\left\langle L_{)(}\right\rangle-\left\langle L_{\asymp}\right\rangle\right) .
$$

If the sign is negative, then $\left\langle L_{)}\right\rangle=0$, and if the sign is positive, then $\left\langle L_{\aleph}\right\rangle=$ $\left\langle L_{)}\right\rangle+\left\langle L_{\asymp}\right\rangle=0$, both of which are contradictions by (3).

Proof of Theorem 1.1 Let $\{L(m)\}$ be an infinite sequence of link diagrams whose Jones polynomials have increasing span and are cyclotomic: For all $m, M(\langle L(m)\rangle)=1$. Suppose that $\{L(m)\}$ has bounded twist number. Passing to a subsequence if necessary, we can assume that all the links are obtained from the same wiring diagram with $N$ twist sites, and since the Jones polynomials change, that these are non-nugatory twist sites. Hence, there is a sequence $\left\{\mathbf{n}(m)=\left(n_{1}(m), \ldots, n_{N}(m)\right)\right\}$ in $\mathbb{Z}^{N}$ such that $L(m)=L_{\mathbf{n}(m)}$. 
Let $|\mathbf{n}(m)|=\sum_{i=1}^{N}\left|n_{i}(m)\right|$, which is the number of crossings of $L_{\mathbf{n}(m)}$. For any $L$, $\operatorname{span}\left(V_{L}(t)\right)$ is a lower bound for the crossing number of $L$, so $|\mathbf{n}(m)| \rightarrow \infty$. Passing to a subsequence if necessary, we can assume that for all $i$, either $n_{i}(m) \rightarrow \infty$, or $n_{i}(m)$ is constant for all $m$. Hence, this subsequence $\{L(m)\}$ is obtained from a fixed diagram $\tilde{L}$ with $k \leq N$ open non-nugatory twist sites at which $n_{i}(m) \rightarrow \infty$. We will call these active twist sites. By Proposition 4.1, to have an infinite sequence of links with distinct cyclotomic Jones polynomials, there must be at least two active twist sites.

Suppose $\{L(m)\}$ has active twist sites for $1 \leq i \leq k$, and $P\left(A, x_{1}, \ldots, x_{k}\right)$ is given by Definition 1. By Proposition 2.1, $V_{L_{\mathbf{n}(m)}}(t)$ is determined by setting $x_{i}=\left(-A^{-4}\right)^{n_{i}}$. Since $4 \operatorname{span}\left(V_{L}(t)\right)=\operatorname{span}(\langle L\rangle)$, our hypothesis that $\operatorname{span}\left(V_{L_{\mathbf{n}(m)}}(t)\right) \rightarrow \infty$ implies

$$
\lim _{m \rightarrow \infty} \operatorname{span}\left(P\left(A, A^{-4 n_{1}(m)}, \ldots, A^{-4 n_{k}(m)}\right)\right)=\infty
$$

Let $\mathbf{x}=\left(x_{1}, \ldots, x_{k}\right), \mathbf{r}=\left(r_{1}, \ldots, r_{k}\right)$, and $\mathbf{x}^{\mathbf{r}}=\prod_{i=1}^{k} x_{i}^{r_{i}}$.

Case 1 Suppose $\lim _{m \rightarrow \infty} v\left(1, n_{1}(m), \ldots, n_{k}(m)\right)=\infty$. By Lemma 3.3:

$$
\begin{aligned}
M\left(P\left(A, x_{1}, \ldots, x_{k}\right)\right) & =\lim _{m \rightarrow \infty} M\left(P\left(A, A^{-4 n_{1}(m)}, \ldots, A^{-4 n_{k}(m)}\right)\right) \\
& =\lim _{m \rightarrow \infty} M\left(P\left(A,\left(-A^{-4}\right)^{n_{1}(m)}, \ldots,\left(-A^{-4}\right)^{n_{k}(m)}\right)\right) \\
& =\lim _{m \rightarrow \infty} M\left(\left\langle L_{\mathbf{n}(m)}\right\rangle\right)=1
\end{aligned}
$$

using $M(\delta)=1$. Lemma 3.1 now implies:

$$
P\left(A, x_{1}, \ldots, x_{k}\right)=A^{\alpha} \mathbf{x}^{\mathbf{r}_{0}} \prod_{j=1}^{\ell} \phi_{j}\left(A^{\alpha_{j}} \mathbf{x}^{\mathbf{r}_{j}}\right)
$$

By (5), some $\mathbf{r}_{j} \neq 0$. Using Lemma 2.4, evaluate $P\left(\xi, x_{1}, \ldots, x_{k}\right)$ at $\xi=e^{2 \pi i / 6}$ :

$$
\prod_{i=1}^{k} x_{i} \prod_{j=k+1}^{N}\left(-\xi^{-3}\right)^{n_{j}}=\xi^{\alpha} \mathbf{x}^{\mathbf{r}_{0}} \prod_{j=1}^{\ell} \phi_{j}\left(\xi^{\alpha_{j}} \mathbf{x}^{\mathbf{r}_{j}}\right)
$$

This is a contradiction: The right-hand side can be made zero for an appropriate choice of nonzero $x_{i}$ 's, whereas the left-hand side will remain nonzero.

Case 2 Suppose $v\left(1, n_{1}(m), \ldots, n_{k}(m)\right)$ is bounded for all $m$. By Lemma 3.4, after passing to a subsequence, there exists $\mathbf{a}=\left(a_{0}, \ldots, a_{k}\right) \in \mathbb{Z}^{k+1} \backslash\{\boldsymbol{0}\}$ such that $\mathbf{a}$. $\left(1, n_{1}(m), \ldots, n_{k}(m)\right)=0$. Without loss of generality, let $a_{k}>0$. We define:

$$
Q\left(A, x_{1}, \ldots, x_{k-1}\right)=P\left(A^{a_{k}}, x_{1}^{a_{k}}, \ldots, x_{k-1}^{a_{k}}, A^{4 a_{0}} \prod_{i=1}^{k-1} x_{i}^{-a_{i}}\right)
$$


$\mathbf{a} \cdot\left(1, n_{1}(m), \ldots, n_{k}(m)\right)=0$ implies $a_{k} n_{k}(m)=-a_{0}-\sum_{i=1}^{k-1} a_{i} n_{i}(m)$. Thus,

$$
Q\left(A, A^{-4 n_{1}(m)}, \ldots, A^{-4 n_{k-1}(m)}\right)=P\left(A^{a_{k}}, A^{-4 a_{k} n_{1}(m)}, \ldots, A^{-4 a_{k} n_{k}(m)}\right) .
$$

By (5), this implies

$$
\lim _{m \rightarrow \infty} \operatorname{span}\left(Q\left(A, A^{-4 n_{1}(m)}, \ldots, A^{-4 n_{k-1}(m)}\right)\right)=\infty
$$

For any $a \in \mathbb{Z} \backslash 0, M(f(t))=M\left(f\left(t^{a}\right)\right)$, so we have:

$$
\begin{aligned}
M\left(Q\left(A, A^{-4 n_{1}(m)}, \ldots, A^{-4 n_{k-1}(m)}\right)\right) & =M\left(P\left(A^{a_{k}}, A^{-4 a_{k} n_{1}(m)}, \ldots, A^{-4 a_{k} n_{k}(m)}\right)\right) \\
& =M\left(P\left(A, A^{-4 n_{1}(m)}, \ldots, A^{-4 n_{k}(m)}\right)\right)
\end{aligned}
$$

Case 2a Suppose $\lim _{m \rightarrow \infty} v\left(1, n_{1}(m), \ldots, n_{k-1}(m)\right)=\infty$. By Lemma 3.3, the previous equation implies:

$$
\begin{aligned}
M\left(Q\left(A, x_{1}, \ldots, x_{k-1}\right)\right) & =\lim _{m \rightarrow \infty} M\left(Q\left(A, A^{-4 n_{1}(m)}, \ldots, A^{-4 n_{k-1}(m)}\right)\right) \\
& =\lim _{m \rightarrow \infty} M\left(P\left(A, A^{-4 n_{1}(m)}, \ldots, A^{-4 n_{k}(m)}\right)\right) \\
& =\lim _{m \rightarrow \infty} M\left(\left\langle L_{\mathbf{n}(m)}\right\rangle\right)=1
\end{aligned}
$$

Lemma 3.1 now implies:

$$
Q\left(A, x_{1}, \ldots, x_{k-1}\right)=A^{\alpha} \mathbf{x}^{\mathbf{r}_{0}} \prod_{j=1}^{\ell} \phi_{j}\left(A^{\alpha_{j}} \mathbf{x}^{\mathbf{r}_{j}}\right)
$$

By (6), some $\mathbf{r}_{j} \neq 0$. Using Lemma 2.4 , evaluate $Q\left(\xi^{1 / a_{k}}, x_{1}, \ldots, x_{k-1}\right)$ at $\xi=$ $e^{2 \pi i / 6}$, and we obtain the same contradiction as in Case 1 .

Case 2b Suppose $v\left(1, n_{1}(m), \ldots, n_{k-1}(m)\right)$ is bounded for all $m$. As we did at the start of Case 2, we reduce $Q\left(A, x_{1}, \ldots, x_{k-1}\right)$ to obtain a $(k-1)$-variable polynomial. If $v\left(1, n_{1}(m), \ldots, n_{k-2}(m)\right)$ is bounded for all $m$, we again reduce to obtain a $(k-2)$ variable polynomial. Proceeding in a similar manner, we may finally reach $\left(1, n_{1}(m)\right)$ where $n_{1}(m)$ is unbounded, so $\lim _{m \rightarrow \infty} v\left(1, n_{1}(m)\right)=\infty$. Whenever we find $j$ such that $\lim _{m \rightarrow \infty} v\left(1, n_{1}(m), \ldots, n_{k-j}(m)\right)=\infty$, we proceed as in Case 2a to obtain a contradiction.

Example 7 This example demonstrates the reduction method in Case 2 of the proof of Theorem 1.1. For some $P\left(A, x_{1}, x_{2}, x_{3}\right)$ in our context, suppose:

$$
\begin{gathered}
\mathbf{n}(m)=\left(2 m, 3 m^{2}+1,5 m-2\right) \\
M\left(\left\langle L_{\mathbf{n}(m)}\right\rangle\right)=M\left(P\left(A,\left(-A^{-4}\right)^{2 m},\left(-A^{-4}\right)^{3 m^{2}+1},\left(-A^{-4}\right)^{5 m-2}\right)\right)
\end{gathered}
$$


Since $(4,-5,0,2) \cdot\left(1,2 m, 3 m^{2}+1,5 m-2\right)=0$, then $v\left(1,2 m, 3 m^{2}+1,5 m-2\right)$ is bounded for all $m$.

$$
\begin{gathered}
Q\left(A, x_{1}, x_{2}\right)=P\left(A^{2}, x_{1}^{2}, x_{2}^{2}, A^{16} x_{1}^{5}\right) \\
Q\left(A,\left(A^{-4}\right)^{2 m},\left(A^{-4}\right)^{3 m^{2}+1}\right)=P\left(A^{2},\left(A^{-8}\right)^{2 m},\left(A^{-8}\right)^{3 m^{2}+1},\left(A^{-8}\right)^{5 m-2}\right) \\
\text { Since } \lim _{m \rightarrow \infty} v\left(1,2 m, 3 m^{2}+1\right)=\infty: \\
M\left(Q\left(A, x_{1}, x_{2}\right)\right)=\lim _{m \rightarrow \infty} M\left(Q\left(A,\left(A^{-4}\right)^{2 m},\left(A^{-4}\right)^{3 m^{2}+1}\right)\right)=\lim _{m \rightarrow \infty} M\left(\left\langle L_{m}\right\rangle\right)=1
\end{gathered}
$$

\section{References}

[1] D W Boyd, Kronecker's theorem and Lehmer's problem for polynomials in several variables, J. Number Theory 13 (1981) 116-121 MR602452

[2] A Champanerkar, I Kofman, On the Mahler measure of Jones polynomials under twisting, Algebr. Geom. Topol. 5 (2005) 1-22 MR2135542

[3] A Champanerkar, I Kofman, E Patterson, The next simplest hyperbolic knots, J. Knot Theory Ramifications 13 (2004) 965-987 MR2101238

[4] T Kanenobu, Examples on polynomial invariants of knots and links, Math. Ann. 275 (1986) 555-572 MR859330

[5] T Kanenobu, Infinitely many knots with the same polynomial invariant, Proc. Amer. Math. Soc. 97 (1986) 158-162 MR831406

[6] M Lackenby, The volume of hyperbolic alternating link complements, Proc. London Math. Soc. (3) 88 (2004) 204-224 MR2018964 With an appendix by Ian Agol and Dylan Thurston

[7] W M Lawton, A problem of Boyd concerning geometric means of polynomials, J. Number Theory 16 (1983) 356-362 MR707608

[8] W B R Lickorish, An introduction to knot theory, Graduate Texts in Mathematics 175, Springer, New York (1997) MR1472978

[9] A Schinzel, The Mahler measure of polynomials, from: "Number theory and its applications (Ankara, 1996)", Lecture Notes in Pure and Appl. Math. 204, Dekker, New York (1999) 171-183 MR1661667

[10] D Silver, A Stoimenow, S Williams, Euclidean Mahler measure and twisted links, Algebr. Geom. Topol. 6 (2005) 581-602

[11] L Watson, Any tangle extends to non-mutant knots with the same Jones polynomial, to appear in J. Knot Theory Ramifications 
Department of Mathematics and Statistics, University of South Alabama Mobile, AL 36688, USA

Department of Mathematics, College of Staten Island, City University of New York 2800 Victory Boulevard, Staten Island, NY 10314, USA

achampanerkar@jaguar1.usouthal.edu, ikofman@math.csi.cuny.edu

Received: 5 June 2006

Algebraic $\mathcal{B}$ Geometric Topology, Volume 6 (2006) 